\title{
Awake intubation with video-assisted laryngoscope or intubating stylet
}

\author{
Jin-Kyoung Kim \\ Department of Anesthesiology and Pain Medicine, Samsung Medical Center, Sungkyunkwan University School of Medicine, Seoul, Korea
}

For anesthesiologists, it is inevitable to confront with various difficult conditions during mask ventilation and intubation. Therefore, anesthesiologists perform a variety of airway examinations and history taking to anticipate and to overcome demanding situations. Yet, the parameters obtained from such measurements are not satisfactory.

In patients having the conditions that indicate difficult intubation, awake intubation is performed especially when the plausibility of mask ventilation is uncertain. Awake fiberoptic intubation has been adopted for management of anticipated difficult tracheal intubation. However, fiberoptic bronchoscope requires higher cost and longer time to learn the technique.

Recently, laryngoscope or stylet with video system is used in various situations, including difficult intubation. Also, in awake intubation, some cases with video-assisted laryngoscope have been reported.

Successful awake video-assisted laryngoscopic intubation has been performed in patients with limited neck extention and Mallampati grade 4 [1]. Also, Park et al. [2] reported a case of a patient with restricted mouth opening, nearly fixed neck movement and regressed mandible due to scar contracture where intubation was performed with the use of fiberoptic bronchoscopy under GlideScope ${ }^{\circledR}$ video laryngoscope guidance.

Recently, Rosenstock reported that in 93 patients with an anticipated difficult intubation, there is no difference in patients' discomfort, ease of the technique and intubation success rate on the first attempt between the awake video-assisted laryngoscopic intubation and awake fiberoptic intubation [3].

Case reports with awake intubation using video-assisted intubating stylet are still rare.

There is a case report that reported successful awake intubation with video-assisted intubating stylet in a patient with fixed neck due to halo vest and limited mouth opening $(3 \mathrm{~cm})$, in addition to modified Mallampati score grade 4 [4].

Smaller diameter of video-assisted intubating stylet can be applied to patients with more limited mouth opening or loose tooth than video-assisted laryngoscope. Video-assisted intubating stylet provided faster and easier intubations than the video-assisted laryngoscope in patients with limited neck motion [5]. In oral, laryngeal, glottic, supraglottic cancer, cancer bleeding during intubation procedure often interfere with the laryngoscopic view. In such situation, owing to smaller volume than video laryngoscope, the video-assisted intubating stylet is helpful to avoid injuring the mass and to overcome potentially traumatic airway manipulation.

Patient preparation for awake intubation with video-assisted laryngoscope or intubating stylet is same as the preparation of awake fiberoptic intubation: antisialogue, oxygen supplement, topical application and transtracheal injection of local anesthetics. For adequate sedation and analgesia, combination of short acting opioids (eg. remifentanil) and sedatives (eg. propofol, or midazolam) infusion can be administered intravenously.

Video-assisted laryngoscope or video-assisted stylet has an external monitor, and the supervisor can see the anatomy together and educate intubation skills on a case-by-case basis. With sufficient training of stylet usage, the quick and easy to use new instrument, such as video-assisted stylet, is expected to be selected as an alternative awake intubating device instead of the

Corresponding author: Jin-Kyoung Kim, M.D., Ph.D., Department of Anesthesiology and Pain Medicine, Samsung Medical Center, Sungkyunkwan University School of Medicine, 50, Ilwon-dong, Gangnam-gu, Seoul 135-710, Korea. Tel: 82-2-3410-2477, Fax: 82-2-3410-6626, E-mail: jkanekim@gmail.com

(c) This is an open-access article distributed under the terms of the Creative Commons Attribution Non-Commercial License (http:// creativecommons.org/licenses/by-nc/3.0/), which permits unrestricted non-commercial use, distribution, and reproduction in any medium, provided the original work is properly cited. 
flexible fiberscope that requires longer time to learn.

However, the currently used video-assisted laryngoscope and intubating stylet do not have the suction function. Therefore, preparations to prevent oral secretion are more important, and the assistant should help the oral suction of secretion or bleeding. In this respect, it is required to develop video-assisted laryngoscope or intubating stylet with the suction function in the near future.

\section{References}

1. Dimitriou VK, Zogogiannis ID, Liotiri DG. Awake tracheal intubation using the Airtraq ${ }^{\circledR}$ laryngoscope: a case series. Acta Anaesthesiol Scand 2009; 53: 964-7.
2. Park CD, Lee HK, Yim JY, Kang IH. Anesthetic management for a patient with severe mento-sternal contracture: difficult airway and scarce venous access -A case report-. Korean J Anesthesiol 2013; 64: 61-4.

3. Rosenstock CV, Thøgersen B, Afshari A, Christensen AL, Eriksen C, Gätke MR. Awake fiberoptic or awake video laryngoscopic tracheal intubation in patients with anticipated difficult airway management: a randomized clinical trial. Anesthesiology 2012; 116: 1210-6.

4. Cheng WC, Lan CH, Lai HY. The Clarus Video System (Trachway) intubating stylet for awake intubation. Anaesthesia 2011; 66: 117880.

5. Kim JK, Kim JA, Kim CS, Ahn HJ, Yang MK, Choi SJ. Comparison of tracheal intubation with the Airway Scope or Clarus Video System in patients with cervical collars. Anaesthesia 2011; 66: 694-8. 